Measuring and explaining the asymmetry of liquidity

Rajat Tayal and Susan Thomas

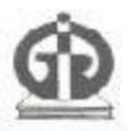

Indira Gandhi Institute of Development Research, Mumbai

March 2012

http://www.igidr.ac.in/pdf/publication/WP-2012-011.pdf 


\title{
Measuring and explaining the asymmetry of liquidity
}

\author{
Rajat Tayal and Susan Thomas \\ Indira Gandhi Institute of Development Research (IGIDR) \\ General Arun Kumar Vaidya Marg \\ Goregaon (E), Mumbai- 400065, INDIA \\ Email (corresponding author): susant@igidr.ac.in
}

\begin{abstract}
This paper examines transactions costs in buying versus selling using a large database of snapshots of the limit order book. On the equity spot market, there is clear evidence of asymmetry in liquidity: transactions costs are lower for buy market orders when compared with sell market orders. In the identical setting, trading in single stock futures is also observed, and there is little evidence of asymmetry. This suggests that asymmetry in liquidity may be driven by short sales restrictions which are present on the spot market but not on the single stock futures market.
\end{abstract}

Keywords: Market microstructure, liquidity asymmetry, Limit Order Book, Spot versus Single Stock Futures, impact cost, liquidity supply schedule.

JEL Code: C53, G10, G12, G18 


\title{
Measuring and explaining the asymmetry of liquidity
}

\author{
Rajat Tayal \\ Susan Thomas* \\ Indira Gandhi Institute of Development Research, Mumbai
}

April 18, 2012

\begin{abstract}
This paper examines transactions costs in buying versus selling using a large database of snapshots of the limit order book. On the equity spot market, there is clear evidence of asymmetry in liquidity: transactions costs are lower for buy market orders when compared with sell market orders. In the identical setting, trading in single stock futures is also observed, and there is little evidence of asymmetry. This suggests that asymmetry in liquidity may be driven by short sales restrictions which are present on the spot market but not on the single stock futures market.
\end{abstract}

${ }^{*}$ The views expressed in this paper belong to the authors and not their employer. We thank Ajay Shah, participants of the IGIDR Finance Research Seminar series, and participants at the Rmetrics workshop at Meielisalp, Switzerland in June 2011, for comments and suggestions to this paper. We are grateful to the National Stock Exchange of India, Ltd., for the data used in this paper. 


\section{Contents}

\begin{tabular}{lll}
\hline 1 & Introduction & 3 \\
\hline
\end{tabular}

2 Asymmetry in transactions costs for buying versus selling $\quad 4$

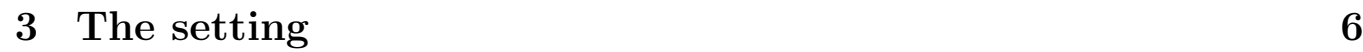

4 Measurement of asymmetry in liquidity in the LOB market 9

4.1 Probability of full market order execution. . . . . . . . . . 11

4.2 Problems of missing data when a market order is not filled . . 11

4.3 A parametric model of the LSS . . . . . . . . . . . . . 14

5 Testing for asymmetry of liquidity in the spot market $\quad 17$

5.1 Evidence on probability of full execution of market orders. . . 19

$5.2 \quad$ Evidence about buy-side versus sell-side IC . . . . . . . . . . . 19

5.3 Evidence about asymmetry from a parametric model of the LSS 19

5.4 Summary . . . . . . . . . . . . . . . . 21

6 Explaining asymmetry: comparing spot versus single stock futures 21

6.1 Evidence on probability of full execution of market orders . . . 23

$6.2 \quad$ Evidence about buy-side versus sell-side IC . . . . . . . . . . 24

6.3 Evidence about asymmetry from a parametric model of the LSS 25

\begin{tabular}{lll}
\hline 7 & Conclusion & 26
\end{tabular} 


\section{Introduction}

The analysis of liquidity in the existing literature has emphasised the bidask spread as a measure of liquidity. The bid-ask spread is a measure of the transactions costs faced when undertaking small transactions, and does not differentiate buying from selling.

In recent decades, the bulk of exchanges worldwide have shifted to the electronic limit order book market. On the electronic limit order book market, the entire limit order book is observed. As a consequence, the instantaneous impact cost faced for placing any market order (either buy or sell) is exactly observed. This makes it possible to reopen the question of asymmetry of liquidity, i.e. the differences between the impact cost in buying versus the impact cost in selling.

In this paper, we analyse a large dataset of snapshots of the limit order book at the National Stock Exchange of India Ltd., one of the world's large electronic limit order book markets. We propose three methods through which we can test for asymmetry between the transactions costs faced in buying versus selling.

On the equity spot market, all three methods suggest that buy-side impact cost (i.e. the transactions costs faced when buying) is lower than sell-side liquidity.

At the same exchange, in the same time zone, using the identical market design, trading in single stock futures takes place in the identical securities. The single stock futures are leveraged cash-settled products. A key difference in the market design lies in a short sales constraints on the spot market: there is no formal mechanism through which shares can be borrowed on the spot market, thus forcing a seller to either own shares ahead of time or borrow them OTC through informal networks for delivery on $\mathrm{T}+2$.

When we repeat the identical empirical analysis for the single stock futures market, we find little evidence of asymmetry. This suggests that restrictions on short sales may be a significant source of asymmetry in liquidity.

The paper thus extends the existing literature on transactions costs in financial markets in two respects. First, we have a pair of findings based on high quality measurement: the null of no asymmetry is clearly rejected for the 
equity spot market and is not rejected for the single stock futures market. Second, implicit in the setting is a suggestive explanation: that short sales constraints may be a significant element to explain asymmetry in liquidity.

The remainder of this paper is organised as follows. Section 2 reviews the existing literature and the potential explanations for asymmetry in liquidity. Section 3 describes the unique setting where our dataset is obtained from. Section 4 proposes three methods through which asymmetry in liquidity can be assessed on a limit order book market. Section 5 applies these three methods to the equity spot market, and finds strong evidence in favour of asymmetry. Section 6 carries the same methods to the single stock futures market, and finds little evidence in favour of asymmetry. Finally, Section 7 summarises the findings and interprets them.

\section{Asymmetry in transactions costs for buy- ing versus selling}

In the analysis of liquidity, one interesting dimension is the asymmetry of liquidity across buy and sell orders. The buy-side of the market is the limit orders of interest to buyers: the sell orders which are available to transact with buyers. Similarly, the sell-side is composed of the waiting limit orders which intend to buy. The asymmetry question consists of exploring differences between transactions costs faced in buying versus transactions costs faced in selling.

The early literature on asymmetry in liquidity - Kraus and Stoll (1972); Chan and Lakonishok (1993); Keim and Madhavan (1996) - attributed liquidity asymmetry to the behaviour of institutions, arguing that purchases have a significant impact on traded prices while traded prices react less when institutions sell. The second strand of literature, Ho and Stoll (1981); Subrahmanyam (1991); Brunnermeier and Pedersen (2009), focused on asymmetry of liquidity arising from the incentives of the NYSE specialist. Since holding inventory is costly, the market maker is keen to offer relatively easy terms to buyers. In addition, selling to an informed buyer is only an opportunity cost to the specialist. On the other hand, buying from an informed seller can result in tangible losses. For securities with higher information uncertainty, 
these asymmetries would be exacerbated. Michayluk and Neuhauser (2008) demonstrate this effectively using a sample of newly listed internet and technology stocks. These are securities with unprecedently high volatilities, and the paper finds that liquidity asymmetry was indeed greater for this class of stocks.

This early literature was rooted in markets with market makers. In recent decades, the bulk of securities exchanges have moved to the electronic limit order book market. This has two implications for the question of asymmetry. First, the measurement of buy and sell impact cost is easily done on a limit order book market, given the full observability of the limit order book. This dimension is the foundation of the present research. Second, a new class of models and explanations is required in this setting, given the absence of designated market makers. As an example, the second strand of literature mentioned above is not relevant when there are no specialists.

One of the initial papers examining asymmetry in order flow was Hedvall et al. (1997), which presented the LOB order flow as a set of demand and supply curves for the buy and sell-side, and showed that the general buy and sell side price impact was likely to be symmetric in these markets, but that it could be asymmetric for specific categories of trades. A more detailed effort is Rosu (2009) which moves away from the information asymmetry assumption of Kyle (1985) and explicitly models different types of orders, multiple agents with the ability to place and cancel multiple orders at various points in time. The paper then derives a series of implications about the dynamic behaviour of the bid-ask spread, price impact of transactions and, most importantly, the evolution of the entire LOB over time. However, while the paper does derive a set of possible forms of the limit order book, it does not address the question of whether there is asymmetry in the liquidity for the buy and sell-side.

The current work is located in the field of analysis of liquidity in the LOB market. In contrast with the papers in the literature which have tried to reconstruct estimates of price impact using the time-series of quotes or traded prices, we observe the entire limit order book, thus permitting direct measurement of impact cost when buying or selling. Unlike the situation in many other exchanges, a large fraction of the activity of the market that we observe comes from retail investors. If asymmetry of liquidity is observed on this market, explanations based on the behaviour of institutional investors 


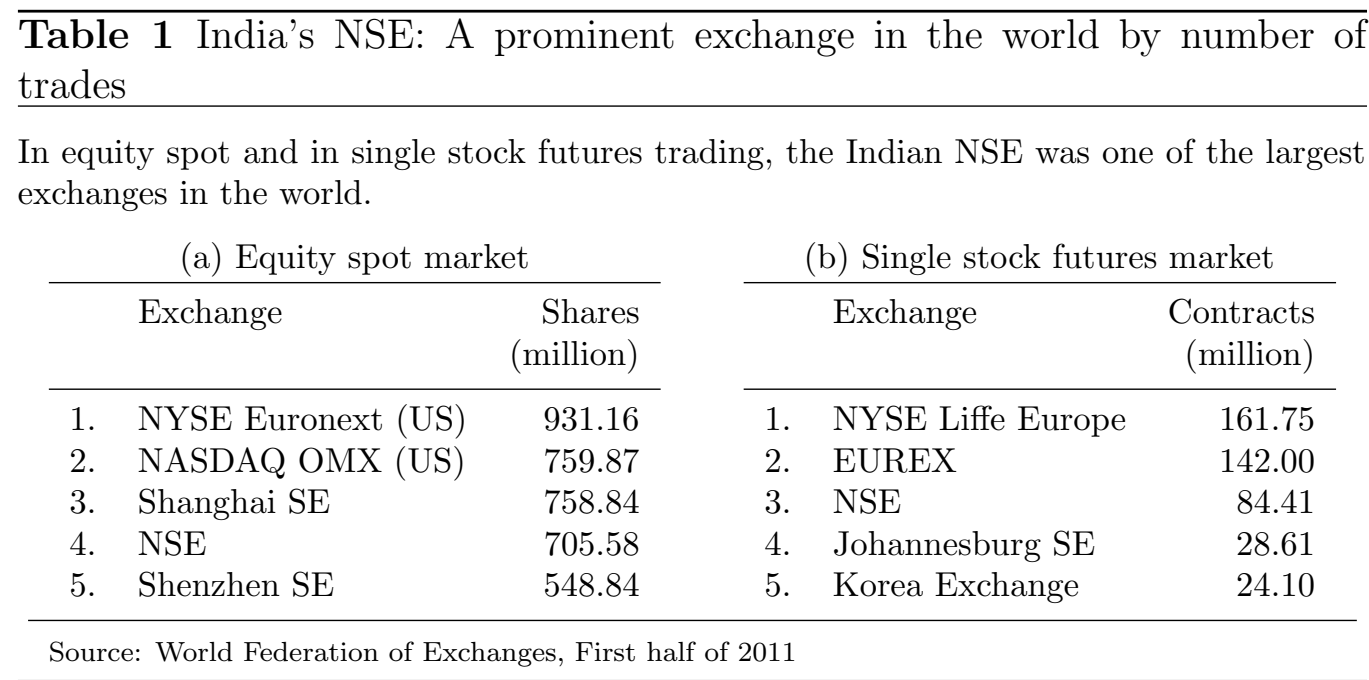

(as proposed in the early literature) are unlikely to be an important source. We explore the role of short sale restrictions in inducing asymmetry of liquidity. The role of short sale restrictions is considered to exacerbate the effect of the information asymmetry (Miller, 1977). Brennan et al. (2010) argue that because short sales is costly, and the demand for illiquidity is likely to be higher for sellers, the premium for illiquidity is likely to be more associated with sell orders. Nguyen et al. (2010) argue that short sales constraints influence the intensity of divergence of opinion among investors, which in turn influence how traders provide liquidity in LOB markets.

In our attempts at understanding financial markets and transactions costs, systematic differences between the costs faced when buying versus selling are an important feature of the modern trading landscape. The existing literature has not arrived at a definite answer on whether asymmetry in liquidity exists and the causes underlying this. This paper sheds light on both questions.

\section{The setting}

The National Stock Exchange (NSE), in India, is one of the most active exchanges in the world by way of equity and derivatives trading. Table 1 
shows that NSE is the 4th largest exchange by the number of shares traded on the equity spot market, and the 3rd largest exchange in the world by the number of contracts traded of stock futures futures. All trading on the exchange is done through an anonymous open electronic limit order book. At any given point in time, in real-time, the order book upto the best five market prices and quantities are visible to all traders. Moreover, on a monthly basis, the exchange publishes archives of the entire limit order book (LOB) as snapshots taken at four different times of the day: 11 A.M., 12 P.M., 1 P.M. and 2 P.M. This rich dataset becomes the source to directly measure liquidity provided in the market.

The NSE is a pure limit order book market. There is no specialist or market maker of any kind. Hence, the evidence obtained from the analysis on NSE fits into the literature on electronic limit order book markets, which have come to dominate the world of trading and exchanges, as opposed to the traditional treatment which emphasised market makers or specialists.

The early literature on the asymmetry of liquidity has emphasised the behaviour of institutional investors as a source of asymmetry. The evidence about NSE is interesting insofar as in the time period used in this paper, 2009 , only 15 percent of the daily turnover on the equity spot market and 20 percent of the turnover on the stock futures market came from institutional investors. ${ }^{1}$ The NSE is a predominantly retail market. Hence, the importance of explanations based on the behaviour of institutional investors is limited.

We focus on the liquidity of 100 securities that trade on the NSE, which that are the largest by market capitalisation. All the snapshots of the LOB taken together in 2009 yields 972 "observations" of the equity spot market LOB and 1,944 including the LOB for the stock futures market. If each order book snapshot for each traded security is counted separately, our dataset comprises 194,400 LOB snapshots.

Table 2 presents summary statistics about spot market liquidity. These include traditional measures of liquidity such as the bid-ask spread and depth for the buy-side and sell-side of the market separately. Each value is the mean of liquidity for the overall and the size-based quintiles, with the stan-

\footnotetext{
${ }^{1}$ Information available on the website of the securities markets regulator, the Securities and Exchanges Board of India, (SEBI) http://www.sebi.gov.in
} 


\section{Table 2 Summary statistics of spot market liquidity}

The table presents summary statistics for liquidity of the sample securities. The statistics are presented for both the overall sample as well as subsets of firms categorised in size quintiles, from S-big (largest market capitalisations) to $S$-small (smallest).

The bid-ask spread is the relative spread, measured as the ratio of the spread as a percentage of the mid-quote price. The inside depth is the sum of the quantities available for trading at the bid and the ask, measured as number of shares. The buy (sell) side depth is the total number of shares available for buying (selling).

For each security, the median value is computed across all order book snapshots. Across all securities in a given category, the sample mean of the medians is reported. The crosssectional standard deviation (of the medians) is presented in parentheses.

\begin{tabular}{lrrccc}
\hline & Market cap. & $\begin{array}{c}\text { Bid-ask } \\
\text { spread }\end{array}$ & $\begin{array}{c}\text { Inside } \\
\text { depth }\end{array}$ & $\begin{array}{c}\text { Sell-side } \\
\text { depth }\end{array}$ & $\begin{array}{c}\text { Buy-side } \\
\text { depth }\end{array}$ \\
\cline { 5 - 6 } & (Rs. billion) & $(\%)$ & \multicolumn{3}{c}{ (Number of shares) } \\
\hline Overall & 97.32 & 0.15 & 4270 & 254700 & 392100 \\
sample & $(332.01)$ & $(0.04)$ & $(11120)$ & $(384290)$ & $(711410)$ \\
\hline S-big & 516.72 & 0.11 & 1670 & 217550 & 272190 \\
& $(473.09)$ & $(0.02)$ & $(1480)$ & $(130290)$ & $(185240)$ \\
S2 & 164.30 & 0.13 & 1930 & 204710 & 269840 \\
& $(53.82)$ & $(0.02)$ & $(1840)$ & $(161010)$ & $(237250)$ \\
S3 & 97.37 & 0.16 & 3440 & 285180 & 463270 \\
& $(13.30)$ & $(0.04)$ & $(6930)$ & $(492720)$ & $(897420)$ \\
S4 & 60.61 & 0.18 & 3600 & 330730 & 577400 \\
& $(12.22)$ & $(0.03)$ & $(5810)$ & $(591150)$ & $(1083280)$ \\
S-small & 32.43 & 0.20 & 10580 & 233460 & 371810 \\
& $(8.72)$ & $(0.03)$ & $(22250)$ & $(340490)$ & $(686130)$ \\
& & & & &
\end{tabular}


dard deviation in paranthesis showing the variation of the means within each sample.

The bid-ask spread shows a systemic increase from the larger market capitalisation firms to the smaller ones, showing that the liquidity provided is higher for the larger compared to the smaller firms, with the smallest firms in the sample showing around double of the spread of the largest firm. Similarly, we would expect that there would be a larger depth for the larger firms and lower depth for the smaller firms. However, this is not observed. A possible explanation is that the number of shares does not reflect the true liquidity available in terms of the value of the transaction. Smaller firms may show a higher depth in terms of shares, but the value of the transaction may be small driven by lower prices on average.

Given our interest in asymmetry of liquidity, it is interesting to see that in Table 2, the depth on the sell-side is consistently lower than the depth on the buy-side. This is a first glimmer of asymmetry of liquidity in the LOB market for the equity spot market.

\section{Measurement of asymmetry in liquidity in the LOB market}

From the full limit order book, it is possible to estimate the exact price $P_{Q}$ that would be paid for a market order of size $Q$. The degree of illiquidity of the security would depend upon how far $P_{Q}$ is from the mid-point quote, $\bar{P}=($ bid + ask $) / 2$. The percentage degradation of $P_{Q}$ compared with $\bar{P}$ is called the impact cost (IC) of the trade.

Thus, for any given transaction size $Q$, each LOB snapshot would yield an estimate of the impact cost for a market order of size $Q$ :

$$
\mathrm{IC}_{q}=100\left(P_{q}-\bar{P}\right) / \bar{P}
$$

When the price impact is calculated for all trade sizes from $Q=1 \ldots \bar{Q}_{\max }$, the entire liquidity supply schedule (LSS) can be traced out, which describes 
Figure 1 The liquidity supply schedule: An illustration

This figure shows one example of the liquidity supply schedule, for shares of Infosys Technologies, at 12 noon on $8^{\text {th }}$ June 2009.

On the y-axis is the set of estimated impact cost of transaction size $Q$. Here, price impact is positive for buy limit orders. The larger the order to buy, the higher the price. As an example, a market order to buy 100,000 shares would require a market impact cost of $1.8 \%$.

On the sell side, price impact is negative for sell limit orders with the sellers obtaining less than the midquote price.

In this example, there is asymmetry in liquidity for all quantities from 70,000 to 150,000 shares: the impact cost in buying appears to be smaller than impact cost in selling.

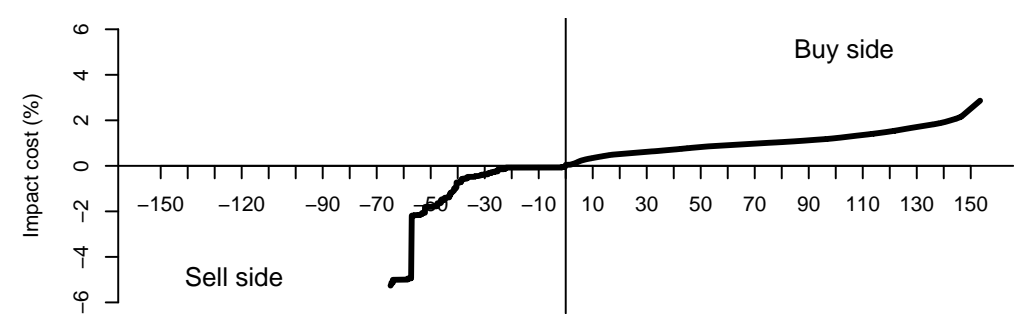

Order size (in '000 shares)

the impact cost faced for all possible buy or sell orders..$^{2}$ In our dataset, the LSS is observed for all securities at all order book snapshots.

An example of the $\mathrm{LSS}_{\text {sell }}$ and $\mathrm{LSS}_{\text {buy }}$ of the LOB for a given security can be seen in Figure 1. We see that the price impact or LSS is a weakly monotonic function in $Q$.

Three measures of liquidity are utilised, in this paper, to obtain evidence about the asymmetry of liquidity between sell-side and buy-side in the electronic limit order book market:

- Probability of full execution of an order of size $Q$, for a fixed set of $Q$.

- Difference in the estimated impact cost to buy versus to sell $\left(\mathrm{IC}_{(\mathrm{sell}, Q)}\right.$ $\left.\mathrm{IC}_{(\mathrm{buy}, Q)}\right)$

\footnotetext{
${ }^{2}$ This graph can be related to the what Chacko et al. (2008) terms the 'quantity structure of immediacy prices'.
} 
- Difference in the estimated parameters of a function fit to the LSS for a given security on the sell-side versus the buy-side.

Each of these measures is discussed in the subsequent three subsections.

\subsection{Probability of full market order execution}

Given the full set of orders, we can calculate whether a single market order of size $Q$ can be fully accomodated by the LOB or not. If the observed depth in the LOB is less than the size of the market order, the order cannot be fully executed. This is a simple measure, which can be measured for any given order size $Q$ for any security. It can be calculated separately for the sell-side and the buy-side of an LOB market.

When a security is observed many times, across different snapshots of the limit order book, we can calculate the probability of complete order execution for an order of size $Q$ by analysing the feasibility of buying or selling on all these order books. If liquidity is symmetric, then for a given $Q$, there should be an equal probability of full market order execution on the sell-side of the LOB as well as the buy-side.

\subsection{Problems of missing data when a market order is not filled}

As discussed above, on the limit order book market, it is possible to measure the buy-side and sell-side impact cost associated with an order of size $Q$ across all order book snapshots. Combining information across multiple order book snapshots, requires addressing special problems of missing data. In order to assess the problems faced, we analyse our data for four order sizes:

- Rs.25,000 - which is the average size of trade on the equity spot market,

- Rs.250,000 - which is the average size of trade on the derivatives market,

- Rs.1 million and Rs.10 million - which corresponds to large orders. 
Table 3 Probability of complete execution of market orders, spot market

The table presents the mean probability of full execution for a few transaction sizes, in size quintiles from $S$-big to $S$-small. The probability of execution is the fraction of limit order book snapshots for which full execution is obtained for a market order of the stated size.

The transaction sizes for which the probability of full execution is calculated are Rs.25,000, Rs.250,000, Rs.1,000,000, and Rs.10 million.

The values in the table are the average value of the probability of full execution for both the buy-side and the sell-side across securities within a quintile. The standard deviation of the averages is reported in parenthesis.

\begin{tabular}{lcccc}
\hline \multirow{2}{*}{$Q$ (in Rs. million) } & \multicolumn{5}{c}{ Probability of full execution } \\
\cline { 2 - 5 } & 0.025 & 0.25 & 1 & 10 \\
\hline Overall & 1.00 & 0.95 & 0.84 & 0.68 \\
sample & $(0.00)$ & $(0.05)$ & $(0.08)$ & $(0.30)$ \\
\hline S-big & 1.00 & 1.00 & 0.95 & 0.84 \\
& $(0.00)$ & $(0.00)$ & $(0.05)$ & $(0.11)$ \\
S2 & 1.00 & 1.00 & 0.92 & 0.76 \\
& $(0.00)$ & $(0.00)$ & $(0.00)$ & $(0.13)$ \\
S3 & 1.00 & 0.93 & 0.88 & 0.68 \\
& $(0.00)$ & $(0.04)$ & $(0.08)$ & $(0.16)$ \\
S4 & 1.00 & 0.90 & 0.83 & 0.61 \\
& $(0.00)$ & $(0.02)$ & $(0.03)$ & $(0.23)$ \\
S-small & 1.00 & 0.80 & 0.72 & 0.49 \\
& $(0.00)$ & $(0.00)$ & $(0.02)$ & $(0.23)$
\end{tabular}


Table 3 shows the fraction of times that a trader would have been able to execute a market order of size $Q$ across the numerous order book snapshots of the dataset. The table shows that only a market order of $Q=$ Rs. 25,000 can be fully executed for all securities in the sample, for all available LOB snapshots.

The table also presents evidence of heterogeneity of the probability of full execution of market orders for firms of different size. From group S-big to group $S$-small the probability of full execution of large order sizes decreases as $Q$ becomes larger. S-big firms LOB have the ability to accomodate full execution of larger orders, going from an average of 95 percent average probability of execution for market orders of Rs.1 million down to 84 percent for an order ten times larger. In comparison, the smallest 20 firms in the sample, show only 72 percent and a mere 49 percent for orders of Rs. 1 and 10 million respectively being filled completely.

When full order execution of a given size is less than certain, this has important implications for the statistical analysis. Consider $\mathrm{IC}_{Q}$ for a given security, which is observed across many order book snapshots, subject to the problem that in certain order book snapshots, a single market order of size $Q$ could not be executed. This induces missing data. At the same time, this missing data represents non-random censoring: IC will be unobserved when there is illiquidity, i.e. when the cost of transacting is very high. Hence, a sample mean computed using only observed values will induce a downward bias. The sample mean of $\mathrm{IC}_{Q}$ computed in this fashion is a biased estimator.

There are two paths through which this can be addressed:

A location estimator of impact cost Suppose $\mathrm{IC}_{Q}$ is observed for more than half of the order book snapshots (with failure in execution for the remainder). We assume that the true IC for unobserved values is a large number. The sample median then constitutes a good location estimator of $\mathrm{IC}_{Q}$. It is insensitive to the specific value adopted for missing data. As long as the basic idea (that missing values correspond to a large IC) is correct, the sample median is a sound location estimator.

As an example, suppose there are five order book snapshots, and the IC values observed for a 1000-share buy order are $(0.5,0.6,0.7, \mathrm{NA}, \mathrm{NA})$ where the last two observations are missing because the order book was not able to support a single buy order for 1000 shares. The sample 
mean of non-missing data, 0.6, is biased downwards since liquidity was poor in the two snapshots where IC was unobserved. If the two NAs are viewed as large values, the sample median -0.7 - is a sound location estimator.

An estimator of the gap between buy and sell impact cost We can observe liquidity asymmetry using the differences of $\mathrm{IC}_{Q}$ for the buyside and the sell-side, subject to the requirement that both these are observed. Here, for a given security $i$, the asymmetry is denoted as $\mathrm{dIC}_{(Q, i)}$, and is measured as:

$$
\mathrm{dIC}_{(Q, i)}=\mathrm{IC}_{(\text {sell-side }, Q, i)}-\mathrm{IC}_{(\text {buy-side }, Q, i)}
$$

dIC is only observed when both buy and sell IC are observed; the sample mean of dIC is then uncontaminated by missing data.

Both these paths are immune to the problem of missing data.

\subsection{A parametric model of the LSS}

The non-parameteric approach has consisted of an examination of a few points on the liquidity supply schedule, and about the depth present in the book. Alternatively, the LSS can be modelled by a parametric function as follows:

$$
\mathrm{IC}_{\text {sell/buy,Q }}=f\left(Q_{\text {sell/buy }}\right)
$$

where $\mathrm{IC}_{\text {sell/buy,Q }}$ is the price impact of a market order to sell or buy $Q$ quantity of the security. Theoretical models of the price impact cost have been proposed to describe the form of the LSS functions, but there has been little consensus so far. Kyle (1985) assumed that impact is both linear in the traded volume and permanent in time.

Bertimas and Lo (1998) assumed a linear permanent price impact while deriving dynamic optimal trading strategies that minimise the expected cost of trading $Q$ over a fixed time horizon. Kempf and Korn (1999) modeled the price impact using a neural network model and found a non-linear relation between net order flow and price changes. Gatheral (2010) assume a no 
dynamic arbitrage principle which implies that the expected cost of trading should be non-negative so that price manipulation is not possible.

Empirical studies broadly conclude that the price impact of trades is an increasing, concave function of trade size $(\sqrt{Q})$ (Evans and Lyons, 2002; Gabaix et al., 2003; Hasbrouck, 1991; Kempf and Korn, 1999; Plerou et al., 2002; Potters and Bouchaud, 2003). A minority of recent studies find no significant deviation from linearity (Engle and Lange, 2001; Breen et al., 2002; Korajczyk and Sadka, 2004). Almgren et al. (2005)) rejects the common square root model in favour of a 3/5 power law function across the range of trade sizes considered. Ting and Warachka (2003) and (Huang and Ting, 2008) use intraday trade data to find support for a S-curve model as best capturing liquidity supply curves in terms of parameter t-statistics and adjusted $R^{2}$ performance. Most recently, Rosu (2009) starts from a structural model where agents place orders into the market continuously, and show that the shape of the LSS can vary between a quadratic and an exponential, or a mixture of the two.

A limitation of the existing literature lies in the use of trade data to estimate price impact and its relation to trade size. In comparison, this paper uses the data observed on the orders placed in the LOB to directly calculate the price impact of the market order at any stated trade size $Q$. This offers a rich dataset for estimation of functions capturing the LSS for any given security.

Once an empirical form for the LSS is estimated, then testing for asymmetry can be carried out by comparing estimated parameter values. Similarly, the impact of short sales constraints on liquidity can be done by calculating the difference between the parameters of the buy-side and sell-side LSS functions for the spot market, and then testing whether there is a comparable difference of parameters for the SSF market or not.

The following models have been identified in the literature in their capacity to represent the LSS:

1. Linear polynomial: $\mathrm{IC}_{Q}=\alpha+\beta Q$

2. Quadratic polynomial : $\mathrm{IC}_{Q}=\alpha+\beta Q+\gamma Q^{2}$

3. Exponential : $\mathrm{IC}_{Q}=\exp ^{\alpha+\beta Q}$

4. Stretched exponential : $\mathrm{IC}_{Q}=\exp ^{\left(\alpha+\beta Q+\gamma Q^{2}\right)}$ 


\section{Table 4 Adjusted $R^{2}$ of alternate functions for the spot market LSS}

The table gives the values of the adjusted $R^{2}$ of the regression for the functional candidates for the bid and the ask side of the limit order book.

Model1 is the linear model. Model2 is the quadratic model. Model3 is the exponential model. Model4 is the stretched exponential model.

The values in the table are the average adjusted $R^{2}$ values for securities in each quintile with the standard deviation of the average presented in paranthesis below. S-big has the securities with the highest market capitalisation securities and $S$-small those with the lowest market capitalisation.

The values in boldface represents the models which have the best fit in terms of adjusted $R^{2}$.

\begin{tabular}{|c|c|c|c|c|c|c|c|c|}
\hline & \multicolumn{4}{|c|}{ Sell side } & \multicolumn{4}{|c|}{ Buy side } \\
\hline & Model1 & Model2 & Model3 & Model4 & Model1 & Model2 & Model3 & Model4 \\
\hline S-big & $\begin{array}{c}0.53 \\
(0.16)\end{array}$ & $\begin{array}{c}0.81 \\
(0.13)\end{array}$ & $\begin{array}{c}0.85 \\
(0.13)\end{array}$ & $\begin{array}{c}\mathbf{0 . 9 0} \\
(0.06)\end{array}$ & $\begin{array}{c}0.51 \\
(0.16)\end{array}$ & $\begin{array}{c}0.79 \\
(0.10)\end{array}$ & $\begin{array}{c}0.85 \\
(0.12)\end{array}$ & $\begin{array}{c}\mathbf{0 . 9 8} \\
(0.05)\end{array}$ \\
\hline$S 2$ & $\begin{array}{c}0.54 \\
(0.13)\end{array}$ & $\begin{array}{c}0.80 \\
(0.10)\end{array}$ & $\begin{array}{c}0.88 \\
(0.09)\end{array}$ & $\begin{array}{c}\mathbf{0 . 9 7} \\
(0.03)\end{array}$ & $\begin{array}{c}0.59 \\
(0.14)\end{array}$ & $\begin{array}{c}0.80 \\
(0.11)\end{array}$ & $\begin{array}{c}0.90 \\
(0.08)\end{array}$ & $\begin{array}{c}\mathbf{0 . 9 1} \\
(0.03)\end{array}$ \\
\hline S3 & $\begin{array}{c}0.57 \\
(0.13)\end{array}$ & $\begin{array}{c}0.83 \\
(0.10)\end{array}$ & $\begin{array}{c}0.88 \\
(0.10)\end{array}$ & $\begin{array}{c}\mathbf{0 . 9 7} \\
(0.04)\end{array}$ & $\begin{array}{c}0.59 \\
(0.13)\end{array}$ & $\begin{array}{c}0.83 \\
(0.10)\end{array}$ & $\begin{array}{c}0.90 \\
(0.09)\end{array}$ & $\begin{array}{c}\mathbf{0 . 9 0} \\
(0.04)\end{array}$ \\
\hline$S 4$ & $\begin{array}{c}0.57 \\
(0.13)\end{array}$ & $\begin{array}{c}0.84 \\
(0.09)\end{array}$ & $\begin{array}{c}0.89 \\
(0.10)\end{array}$ & $\begin{array}{c}\mathbf{0 . 9 8} \\
(0.04)\end{array}$ & $\begin{array}{c}0.56 \\
(0.13)\end{array}$ & $\begin{array}{c}0.82 \\
(0.10)\end{array}$ & $\begin{array}{c}0.89 \\
(0.09)\end{array}$ & $\begin{array}{c}\mathbf{0 . 9 2} \\
(0.03)\end{array}$ \\
\hline S-small & $\begin{array}{c}0.58 \\
(0.13)\end{array}$ & $\begin{array}{c}0.85 \\
(0.09)\end{array}$ & $\begin{array}{c}0.89 \\
(0.10)\end{array}$ & $\begin{array}{c}\mathbf{0 . 9 7} \\
(0.03)\end{array}$ & $\begin{array}{c}0.57 \\
(0.13)\end{array}$ & $\begin{array}{c}0.83 \\
(0.10)\end{array}$ & $\begin{array}{c}0.90 \\
(0.09)\end{array}$ & $\begin{array}{c}\mathbf{0 . 9 0} \\
(0.03)\end{array}$ \\
\hline
\end{tabular}

Each of these have a common feature of being monotonically increasing in $Q$. Each of these models has an intercept term and one or more slope coefficients, which captures how the price impact cost changes for larger order sizes.

For our empirical analysis, these functions are estimated using all calculated impact cost, $Q$ pairs for the full LOB for every security, separately for the buy-side and the sell-side. For each security, the average adjusted $R^{2}$ is calculated for all the buy-side LOB and the sell-side LOB observations. Then, the average of these adjusted $R^{2}$ are calculated for the sample, which are reported in the table. The model with the highest adjusted $R^{2}$ is chosen as the best representation of the LSS.

The results in Table 4 strongly suggest that the stretched exponential (Model 4 ) is the best model according to the adjusted $R^{2}$. Under this model, 


$$
\frac{\partial \mathrm{IC}}{\partial Q}=(\beta+2 \gamma Q) \mathrm{IC}_{Q}
$$

As $Q$ goes up, IC worsens not just by $\beta$ and $\mathrm{IC}_{Q}$, but also by $\gamma$ and $Q$ itself. Thus, the stretched exponential involves a sharper worsening of IC as $Q$ increases. This, in turn, implies that market liquidity for a security, where the LSS of the security follows a stretched exponential, tends to be more sensitive to changes in $Q$.

The estimated parameter values of this parametric model of the LSS $-\hat{\alpha}, \hat{\beta}, \hat{\gamma}$ - can be used to test for asymmetry, by examining the following hypotheses:

- Is $\hat{\alpha}_{S}>\hat{\alpha}_{B}$ ?

- Is $\hat{\beta}_{S}>\hat{\beta}_{B}$ ?

- Is $\hat{\gamma}_{S} \neq \hat{\gamma}_{B}$ ?

If the estimated sell-side parameters are individually and jointly lower/higher than the estimated buy side parameters, we would conclude that the sell-side liquidity is better/worse than buy-side liquidity. We use the KolmogorovSmirnov (KS) test on the distributions of estimated $\alpha_{B}, \alpha_{S}, \beta_{B}, \beta_{S}, \gamma_{B}, \gamma_{S}$ to establish whether there are asymmetries in liquidity. Further, we can also test for the direction in the asymmetries: where the estimated parameters tend to be lower on the buy-side compared to the sell-side, which would support the hypothesis that the liquidity provided in the LOB indicates the fear of asymmetric information by the liquidity providers.

\section{Testing for asymmetry of liquidity in the spot market}

We first present results of our analysis, through the three different testing procedures, for the equity spot market. 


\section{Table 5 Probability of full execution on spot LOB}

In the dataset, a large number of snapshots of the limit order book are observed. For each security, in each snapshot, a single market order of size $Q$ is attempted. Some order books are unable to support a market order of this size. Based on this, we compute the fraction of order books for which the transaction could be completed.

This probability of full execution is showed in each of the size quintiles, going from S-big (the biggest) to $S$-small (the smallest). Values in boldface indicate that the probability of execution on one side of the book is statistically higher at a $5 \%$ level of significance.

As an example, this tells us that amongst the smallest quintile of stocks, a single buy order for Rs. 1 million is executed with a 0.72 per cent probability when it is a sell order, but a statistically significantly higher probability of 0.80 when it is a buy order.

For all firm sizes, for large sized orders, there is a higher probability of being able to place a single large order on the buy side. This shows the presence of asymmetry between buying and selling; sell side liquidity is worse than buy side liquidity.

\begin{tabular}{|c|c|c|c|c|c|c|c|c|}
\hline & \multicolumn{4}{|c|}{ Sell-side $Q$ (Rs. Mln.) } & \multicolumn{4}{|c|}{ Buy-side $Q$ (Rs. Mln.) } \\
\hline & 0.025 & 0.25 & 1 & 10 & 0.025 & 0.25 & 1 & 10 \\
\hline$S$-big & 1 & 1 & 0.90 & 0.78 & 1 & 1 & 0.98 & 0.91 \\
\hline$S 2$ & 1 & 1 & 0.88 & 0.71 & 1 & 1 & 0.96 & 0.82 \\
\hline S3 & 1 & 0.90 & 0.80 & 0.65 & 1 & 0.96 & 0.90 & 0.70 \\
\hline S4 & 1 & 0.88 & 0.80 & 0.50 & 1 & 0.92 & 0.86 & 0.62 \\
\hline S-small & 1 & 0.80 & 0.72 & 0.39 & 1 & 0.87 & 0.80 & 0.55 \\
\hline Overall sample & 1 & 0.92 & 0.78 & 0.27 & 1 & 1 & 0.84 & 0.40 \\
\hline
\end{tabular}




\subsection{Evidence on probability of full execution of market orders}

Table 5 shows that there is a higher probability of executing large orders on the buy-side compared to the sell-side. It is easier to buy large amounts of shares rather than to sell large amounts of shares, and is one indicator of asymmetry in the market liquidity. The evidence in favour of asymmetry is striking: all the cells in the table, with values other than 1 , have statistically significant differences between buying and selling.

\subsection{Evidence about buy-side versus sell-side IC}

Table 6 present the average $\mathrm{dIC}_{Q}$ for a given sample of securities, where $\mathrm{dIC}_{(Q, i)}$ is calculated as the median value of the difference between sell-side IC and buy-side IC for all the LOB observations of a security $i$. The standard deviation of the sample average is reported in parenthesis as well. The results show that for small $Q$, liquidity is symmetric for buyers and sellers.

However, as $Q$ becomes larger, $\mathrm{dIC}_{(Q, i)}$ becomes positive and significant. This implies that it is more difficult to sell large quantities than it is to buy the same $Q$. For example, in the overall sample, $\mathrm{IC}_{\text {sell }}$ for $Q \geq \mathrm{Rs} .1$ million is, on average, 1.5 times higher than the value of $\mathrm{IC}_{\mathrm{buy}}$ for the same $Q$.

The evidence for the behaviour of liquidity asymmetry persists across all quartiles of securities by market capitalisation. For small $Q$, buy-side and sell-side liquidity is symmetric, and asymmetric for larger $Q$ with a larger premium on the sell-side.

\subsection{Evidence about asymmetry from a parametric model of the LSS}

Our liquidity measures here are the parameter estimates of the stretched exponential model, which are the parameter $\alpha$, which expresses the base level of liquidity and the parameters $\beta$ and $\gamma$, which express the rate of change of liquidity with $Q$. 
Table 6 Average difference between buy-side and sell-side liquidity in the spot market

In the dataset, a large number of snapshots of the limit order book are observed. For each security, in each snapshot, a single market order of size $Q$ is attempted. We discard snapshots where a full execution was not obtained for either buy or sell. For the remainder, we compute $\mathrm{dIC}_{(Q, i)}=\mathrm{IC}_{(\text {sell-side, },, i)}-\mathrm{IC}_{(\text {buy-side, },, i)}$, the extent to which sell impact cost (in per cent) is bigger than buy impact cost (in per cent).

For each security, the median value across multiple snapshots is utilised. Each cell of the table shows the sample mean of the values across all securities. The values in brackets are sample standard deviations. The values in boldface indicate instances when $\mathrm{dIC}_{(Q)}$ are different from zero and statistically significant at a 95 per cent level. In these values, sell side liquidity is worse than buy side liquidity.

As an example, this shows us that in the smallest quintile of firms, for transactions of Rs.1 million, on average, sell impact cost was worse than buy impact cost by 0.39 percentage points. This difference was statistically significant at a 95 per cent level.

\begin{tabular}{lrrrr}
\hline & \multicolumn{4}{c}{$Q$ (Rs. Mln.) } \\
\cline { 2 - 5 } & 0.025 & 0.25 & 1 & 10 \\
\hline S-big & 0.01 & 0.02 & 0.04 & $\mathbf{0 . 5 2}$ \\
& $(0.00)$ & $(0.03)$ & $(0.12)$ & $(0.81)$ \\
S2 & 0.01 & 0.04 & 0.04 & $\mathbf{0 . 7 8}$ \\
& $(0.01)$ & $(0.06)$ & $(0.25)$ & $(1.37)$ \\
S3 & 0.02 & 0.09 & 0.10 & $\mathbf{1 . 2 0}$ \\
& $(0.02)$ & $(0.15)$ & $(0.50)$ & $(1.23)$ \\
S4 & 0.02 & 0.11 & 0.12 & $\mathbf{0 . 9 7}$ \\
& $(0.01)$ & $(0.09)$ & $(0.29)$ & $(1.92)$ \\
S-small & 0.03 & 0.04 & $\mathbf{0 . 3 9}$ & $\mathbf{2 . 6 8}$ \\
& $(0.02)$ & $(0.27)$ & $(0.61)$ & $(3.36)$ \\
\hline Overall & 0.02 & 0.04 & 0.05 & $\mathbf{0 . 9 5}$ \\
sample & $(0.02)$ & $(0.16)$ & $(0.43)$ & $(1.69)$
\end{tabular}


Table 7 LSS function estimates for the spot market LOB

The values presented are summary statistics of the parameter estimates of the stretched exponential model $-\alpha, \beta, \gamma-$ for the sample.

The table shows average values of the median, minimum and maximum values for the securities in the overall sample, as well as, market capitalisation based quintiles, $S 1-S 5$. $S$-big securities having the highest market capitalisation and $S$-small having the lowest. These parameters are calculated separately for the sell-side and the buy-side.

\begin{tabular}{lrrrrrr}
\hline & $\hat{\alpha}_{S}^{S}$ & $\hat{\alpha}_{B}^{S}$ & $\hat{\beta}_{S}^{S}$ & $\hat{\beta}_{B}^{S}$ & $\hat{\gamma}_{S}^{S}$ & $\hat{\gamma}_{B}^{S}$ \\
\hline S-big & $\mathbf{2 . 9 3}$ & 2.11 & $\mathbf{0 . 1 4}$ & 0.12 & $\mathbf{3 . 4 4}$ & 2.62 \\
$S 2$ & $\mathbf{3 . 5 8}$ & 2.14 & $\mathbf{0 . 1 3}$ & 0.10 & $\mathbf{3 . 1 4}$ & 2.41 \\
$S 3$ & $\mathbf{4 . 6 3}$ & 2.67 & $\mathbf{0 . 1 2}$ & 0.10 & $\mathbf{2 . 9 2}$ & 2.36 \\
$S 4$ & $\mathbf{4 . 2 2}$ & 2.90 & $\mathbf{0 . 3 8}$ & 0.33 & $\mathbf{3 . 2 9}$ & 2.95 \\
S-small & $\mathbf{6 . 5 0}$ & 3.84 & $\mathbf{0 . 6 0}$ & 0.52 & $\mathbf{3 . 3 8}$ & 2.38 \\
\hline Overall & 3.63 & 2.42 & 0.20 & 0.16 & 3.26 & 2.44
\end{tabular}

These estimates are presented in Table 7 as $\hat{\alpha}^{S}, \hat{\beta}^{S}, \hat{\gamma}^{S}$ where the superscript $S$ indicates that these are spot market estimates. All the parameter estimates are positive which is consistent with the observation that liquidity worsens for larger order sizes. The sell-side estimates are consistently higher than the buy-side for the securities in the case of all three parameters. This implies that the drop off in liquidity is worse for sellers compared with buyers in the market.

\subsection{Summary}

We have examined buying and selling on the equity spot market using three different estimation strategies. Across all three methods, there is striking evidence of asymmetry in liquidity: buying is easier than selling.

\section{Explaining asymmetry: comparing spot ver- sus single stock futures}

One potential explanation that could shape differences in liquidity between buying and selling lies in short sale restrictions. These restrictions are in- 
nately asymmetric and thus merit exploration.

In order to assess the role (if any) of short sale restrictions, we exploit a remarkable feature of our setting: the presence of a single stock futures (SSF) market alongside the equity spot market. Trading in equity spot and their related single stock futures for the identical securities, takes place at NSE with the identical market rules. The same trading system is used; trading starts and stops at the same time; trading is undertaken by the identical securities firms; etc.

There is, however, one major difference: the equity spot market features a very strong short sale restriction (the absence of a formal short selling mechanism) $)^{3}$ while the single stock futures (SSF) market is cash settled so there is no difference between long and short positions. The fact that both the SSF (where long and short positions are symmetric) and spot market (where short sales is difficult) trades simultaneously offers an opportunity to understand the extent to which short sales explains asymmetries in liquidity.

In this section, we use the same methodology used to establish asymmetry of liquidity between sell-side and buy-side to analyse liquidity on the SSF markets. We compare these results with that of the spot market in order to highlight the effect of short sales constraints on liquidity asymmetry. If both markets show similar asymmetry in liquidity, we may conclude that short sale restrictions are not an important factor explaining asymmetry in liquidity. At the other extreme, if the SSF market displays no asymmetry in liquidity, we may conclude that the only source of asymmetry in liquidity is short sale restrictions. 
Table 8 Probability of full execution of market orders in the SSF market

The values reported in the table are the fraction of the LOB data for a given security where a market order of size $Q$ gets immediate and complete execution.

This probability of full execution is presented as the average for the overall sample, as well as for size quintiles, going from $S$-big (the biggest) to $S$-small (the smallest). Values in boldface indicate that the probability of execution on one side of the book is statistically higher at a $95 \%$ level of significance.

As an example, among the stocks in the smallest quintile, a single order to sell Rs.1 million has a 98 percent probability of full execution while a buy order has a 99 percent probability of full execution.

\begin{tabular}{|c|c|c|c|c|c|c|c|c|}
\hline & \multicolumn{4}{|c|}{ Sell-side $Q$ (Rs. Mln.) } & \multicolumn{4}{|c|}{ Buy-side $Q$ (Rs. Mln.) } \\
\hline & 0.025 & 0.25 & 1 & 10 & 0.025 & 0.25 & 1 & 10 \\
\hline Overall sample & & 1 & 1 & 0.82 & & 1 & 1 & 0.79 \\
\hline$S$-big & & 1 & 1 & 0.98 & & 1 & 1 & 0.98 \\
\hline$S 2$ & & 1 & 1 & 0.95 & & 1 & 1 & 0.93 \\
\hline$S 3$ & & 1 & 0.99 & 0.82 & & 1 & 1 & 0.81 \\
\hline$S 4$ & & 1 & 1 & 0.74 & & 1 & 1 & 0.70 \\
\hline$S$-small & & 1 & 0.98 & 0.64 & & 1 & 0.99 & 0.55 \\
\hline
\end{tabular}

\subsection{Evidence on probability of full execution of market orders}

Table 8 shows the percentage of times the trader would be able to fully execute a market order on the sell-side and the buy-side of the SSF markets. The comparison of the numbers show that the probability of full execution drops for larger transaction order sizes in the futures market, just as it did in the spot market. Also, the probability of full execution does decrease for SSF of the larger sized (higher capitalised) firms to smaller sized firms. However, this drop off tends to be symmetric for both the spot and the SSF market. There is no cell in the table which is in boldface, i.e. significant at a 95 per cent level of significance.

\footnotetext{
${ }^{3}$ The sell position at the end of each trading day is required to induce delivery of shares on date $T+2$. When speculators have a negative view about a price, they have two choices. One is that they can coincidentally own the stock and thus sell it off. Alternatively, they can borrow shares OTC from informal networks. The absence of a formal stock borrowing / margin trading mechanism (as was the case in India during the period of the study in this paper) amounts to an important restriction on short sales.
} 
Table 9 Average difference between sell-side and buy-side liquidity in the SSF market

The table presents the difference between the $\mathrm{IC}_{(\text {sell-side,Q) }}$ and $\mathrm{IC}_{(\text {buy-side,Q) }}$ on the spot and the SSF market. Snapshots where a full execution was not obtained for either buy or sell were discarded. $\mathrm{dIC}_{(Q, i)}$ is computed as $\mathrm{IC}_{(\text {sell-side,Q,i) }}-\mathrm{IC}_{(\text {buy-side,Q,i) }}$. The median value is calculated for each security, and the avearge is reported for the overall sample as well as for the quartiles by size.

The values in brackets are sample standard deviations. The values in boldface indicate instances when $\mathrm{dIC}_{(Q)}$ are different from zero and statistically significant at a 95 per cent level. For these values, sell side liquidity is worse than buy side liquidity.

\begin{tabular}{cccc}
\hline & \multicolumn{3}{c}{$Q$ (Rs. Mln. } \\
\cline { 2 - 4 } S-big & 0.25 & 1 & 10 \\
& 0.00 & 0.01 & 0.05 \\
S2 & $(0.00)$ & $(0.01)$ & $(0.09)$ \\
& 0.00 & 0.02 & 0.09 \\
S3 & $(0.00)$ & $(0.02)$ & $(0.24)$ \\
& 0.01 & 0.13 & 0.02 \\
S4 & $(0.04)$ & $(0.32)$ & $(0.27)$ \\
& 0.00 & 0.07 & $\mathbf{0 . 7 1}$ \\
& $(0.01)$ & $(0.06)$ & $(1.14)$ \\
S-small & 0.00 & 0.05 & 0.18 \\
& $(0.01)$ & $(0.06)$ & $(0.58)$ \\
\hline Overall & 0.00 & 0.06 & 0.14 \\
sample & $(0.02)$ & $(0.15)$ & $(0.61)$
\end{tabular}

This first block of evidence shows that the SSF market has no asymmetry in liquidity.

\subsection{Evidence about buy-side versus sell-side IC}

In order to test whether there is asymmetry in the price impact of market orders in the SSF market, $\mathrm{dIC}_{Q, i}$ is calculated using the SSF market LOB for the same range of $Q$ sizes of Rs.0.25,1,10 million. Table 9 shows the median IC calculated across all observations, for the overall sample as well 
Table 10 LSS parameter estimates for the SSF market LOB

The values presented are summary statistics of the parameter estimates of the stretched exponential model $-\alpha, \beta, \gamma$ - fit to the LSS data from the SSF markets for the sample. The table shows average values of the median, minimum and maximum values for the parameters for the overall sample, as well as the market capitalisation based quintiles, $S 1-S 5$. S-big securities having the highest market capitalisation and $S$-small having the lowest. These parameters are calculated separately for the sell-side and the buy-side.

\begin{tabular}{l|cc|cc|cc}
\hline & $\alpha_{\text {sell }}$ & $\alpha_{\text {buy }}$ & $\beta_{\text {sell }}$ & $\beta_{\text {buy }}$ & $\gamma_{\text {sell }}$ & $\gamma_{\text {buy }}$ \\
\hline Overall & 0.72 & $\mathbf{1 . 1 2}$ & $\mathbf{0 . 1 8}$ & 0.12 & $\mathbf{1 . 8 2}$ & 0.84 \\
S-big & 0.76 & $\mathbf{0 . 8 7}$ & $\mathbf{0 . 1 2}$ & 0.08 & $\mathbf{1 . 0 5}$ & 0.73 \\
S2 & 0.87 & $\mathbf{1 . 0 6}$ & $\mathbf{0 . 1 4}$ & 0.08 & $\mathbf{1 . 5 8}$ & 0.84 \\
S3 & 0.64 & $\mathbf{1 . 0 2}$ & $\mathbf{0 . 1 9}$ & 0.11 & $\mathbf{0 . 8 1}$ & 0.79 \\
S4 & 0.74 & $\mathbf{1 . 3 5}$ & $\mathbf{0 . 2 1}$ & 0.12 & $\mathbf{2 . 0 0}$ & 1.63 \\
S-small & 0.43 & $\mathbf{0 . 7 3}$ & $\mathbf{0 . 2 6}$ & 0.15 & $\mathbf{2 . 8 4}$ & 2.64
\end{tabular}

as for quintiles based on size.

In contrast with the results for the spot market, most cells in this table are not in boldface. There is some evidence in favour of asymmetry, but there is little statistical significance. This second block of evidence also supports the idea that on the SSF market, there is a lack of asymmetry in liquidity.

\subsection{Evidence about asymmetry from a parametric model of the LSS}

Lastly, we examine the estimated parameter values for the stretched exponential functions fit to the SSF LOB separately for the buy-side and the sell-side. This is presented in Table 10 as $\hat{\alpha}^{F}, \hat{\beta}^{F}, \hat{\gamma}^{F}$ to denote the parameter estimates for the SSF LSS functions. The values of all the parameter estimates are positive for the SSF LSS. This is consistent with the notion that liquidity (as measured by the IC) worsens as the order size $Q$ becomes larger, and similar to the values estimated for the spot LSS functions.

However, we observe that $\hat{\alpha}_{S}^{F}$ is consistently less than $\hat{\alpha}_{B}^{F}$. This is the opposite to what was observed in the case of the spot market in Table 7 where $\hat{\alpha}_{S}^{S}>$ $\hat{\alpha}_{B}^{S}$. Thus, while the $\hat{\beta}^{F}$ and $\hat{\gamma}^{F}$ parameters are greater for the sell-side compared to the buy-side, the intercept parameter is the opposite. Thus, we 
cannot infer that the parameters support the position that the liquidity on the sell-side is worse than liquidity on the buy-side consistently.

Thus, the overall evidence across the non-parameteric and parameteric measures suggests that there is much lower presence of asymmetry in the liquidity of the SSF markets. While, there is some evidence that liquidity does become skewed on one side compared to the other, there is no evidence of a systematic bias in liquidity between the buy-side and the sell-side LOB in the SSF markets.

\section{Conclusion}

The field of market microstructure is concerned with measuring and understanding liquidity, i.e. the transactions costs faced in trading. The bid-ask spread, which is the workhorse of the bulk of the theoretical and empirical literature, encourages a symmetric treatment of buying versus selling. However, the bulk of exchanges worldwide have now shifted to becoming electronic limit order book markets. On the limit order book market, the bid-ask spread is only a small part of the information set on liquidity, when the entire limit order book is observed. This permits the direct observation of liquidity faced when buying versus selling.

The contribution of this paper lies in high quality empirical evidence about asymmetry in liquidity. The impact cost faced when a single market order is placed is directly observed on a limit order book market, thus eliminating the complexities that arise in trying to infer the cost of transacting under other market structures. We draw on evidence about impact cost on the limit order book, for all transaction sizes, for 194,400 snapshots of the limit order book, on one of the most active exchanges of the world. Based on this, we strongly reject the null of symmetry in liquidity on the spot market. At the same time, we find little evidence of asymmetry on the SSF market.

The empirical work of the paper has approached the question from three perspectives, harnessing a careful treatment of missing data when market orders are not filled, and the selection of a best-fit parametric model of the liquidity supply schedule. The answers obtained through the three methods are broadly consistent, which suggests that the findings are robust. 
The theoretical literature has proposed explanations for asymmetry rooted in the behaviour of market makers, institutional investors, short sales constraints, and asymmetric information. The evidence of this paper does not constitute a decisive hypothesis test about any of these explanations. At the same time, it suggests certain interpretations:

- The NSE is a retail-dominated market, with $15 \%$ of turnover on the spot market coming from institutional investors and $20 \%$ of turnover on the futures market coming from institutional investors. This suggests that explanations based on the behaviour of institutional investors should be relatively unimportant in this setting.

- There are no market makers on the NSE; this suggests that explanations based on the behaviour of market makers would be relatively unimportant in this setting.

- Short sales constraints are strongly present on the spot market and completely absent on the stock futures market.

- To the extent that informed traders prefer to trade on the leveraged market, asymmetric information and adverse selection may be exacerbated on the stock futures market. However, we find little evidence of asymmetry on the SSF market, thus de-emphasising explanations based on asymmetric information and adverse selection.

Our analysis of the stock futures market finds little evidence in favour of asymmetry. This undermines the case for asymmetric information and adverse selection as important factors inducing asymmetry in liquidity. This suggests that the residual explanation - short sales constraints - is important in explaining asymmetry in liquidity. Future analytical work is required which will integrate the leverage of the single stock futures market with the lack of short sale restrictions into a potentially more complex explanation. 


\section{References}

Almgren R, Thum C, Hauptmann E, Li H (2005). "Direct estimation of equity market impact." Journal of Risk, 18, 57.

Bertimas D, Lo AW (1998). "Optimal control of execution costs." Journal of Financial Markets, 1, 1-50.

Breen WJ, Hodrick LS, Korajczyk RA (2002). "Predicting equity liquidity." Management Science, 48, 470-483.

Brennan MJ, Chordia T, Subrahmanyam A, Tong Q (2010). "Sell-Order Liquidity and the Cross-Section of Expected Stock Returns."

Brunnermeier M, Pedersen L (2009). "Market liquidity and funding liquidity." Review of Financial Studies, 22, 2201-2232.

Chacko GC, Jurek JW, Stafford E (2008). "The price of immediacy." Journal of Finance, LXIII(3), 1253-1290.

Chan LKC, Lakonishok J (1993). "Institutional trades and intraday stock price behavior." Journal of Financial Economics, 33(2), 173-199.

Engle RF, Lange J (2001). "Predicting VNET: A model of the dynamics of the market depth." Journal of Financial markets, 4, 113-142.

Evans M, Lyons R (2002). "Order flow and exchange rate dynamics." Journal of Political Economy, 110, 170.

Gabaix X, Gopikrishnan P, Plerou V, Stanley H (2003). "A theory of power-law distributions in financial market fluctuations." Nature, 423, 267.

Gatheral J (2010). "No dynamic arbitrage and market impact." Quantitative Finance, 10, 749 .

Hasbrouck J (1991). "Measuring the information content of stock trades." Journal of Finance, 46, 179-207.

Hedvall K, Niemeyer J, Rosenqvist G (1997). "Do buyers and sellers behave similarly in a limit order book? A high frequency data examination of the Finnish stock exchange." Journal of Empirical Finance, 4(2-3), 279-293.

Ho T, Stoll H (1981). "Optimal dealer pricing under transactions and return uncertainty." Journal of Financial Economics, 9, 47-73. 
Huang RD, Ting C (2008). "A functional approach to the price impact of stock trades and the implied true price." Journal of Empirical Finance, 15, 1-16.

Keim D, Madhavan A (1996). "The upstairs market for large-block transactions: analysis and measurement of price effects." Review of Financial Studies, 9(1), $1-36$.

Kempf A, Korn O (1999). "Market depth and order size." Journal of Financial Markets, 2, 29.

Korajczyk RA, Sadka R (2004). "Are momentum profits robust to trading costs?" Journal of Finance, 59(3), 1039-1082.

Kraus A, Stoll HR (1972). "Price Impacts of Block Trading on the New York Stock Exchange." Journal of Finance, 27(3), 569-588.

Kyle AS (1985). "Continuous auctions and Insider trading." Econometrica, 53(6), $1315-1335$.

Michayluk D, Neuhauser K (2008). "Is Liquidity Symmetric? A Study of Newly Listed Internet and Technology Stocks." International Review of Finance, 8(34), 159-178.

Miller E (1977). "Risk, Uncertainty, and Divergence of Opinion." The Journal of Finance, 32, 1151-1168.

Nguyen AH, Duong HN, Kalev PS, Oh NY (2010). "Implicit Trading Costs, Divergence of Opinion, and Short-Selling Constraints in the Limit Book Order Market." The Journal of Trading, 5(2), 92-101.

Plerou V, Gopikrishnan P, Gabaix X, , Stanley H (2002). "Quantifying stock-price response to demand fluctuations." Physical Review E, 66, 027104.

Potters M, Bouchaud J (2003). "More statistical properties of order books and price impact." Physica A, 324, 133-140.

Rosu I (2009). "A dynamic model of the limit order book." Review of Financial Studies, 22(11), 4601-4641.

Subrahmanyam A (1991). "Risk aversion, market liquidity, and price efficiency." Review of Financial Studies, 4, 417-441.

Ting C, Warachka M (2003). "A new methodology for measuring liquidity induced transaction costs." Working paper, Singapore Management University. 